\title{
Reproductive colonization of land by frogs: embryos and larvae excrete urea to avoid ammonia toxicity
}

\author{
Javier Mendez Narvaez ${ }^{1}$ and Karen Warkentin ${ }^{1}$ \\ ${ }^{1}$ Boston University
}

August 5, 2021

\begin{abstract}
Vertebrate colonization of land occurred multiple times, including over 50 origins of terrestrial eggs in frogs. Some environmental factors and phenotypic responses that facilitated these transitions are known, but responses to water constraints and risk of ammonia toxicity during early development are poorly understood. We tested if ammonia accumulation and dehydration risk induce a shift from ammonia to urea excretion during in early stages of four anurans, from three origins of terrestrial development. We quantified ammonia and urea concentrations during early development on land, under well-hydrated and dry conditions. Where we found urea excretion, we tested for a plastic increase under dry conditions and with ammonia accumulation in developmental environments. We assessed the potential adaptive role of urea excretion by comparing ammonia tolerance measured in 96h-LC50 tests with ammonia levels in developmental environments. Ammonia accumulated in foam nests and perivitelline fluid, increasing over development and reaching higher concentrations under dry conditions. All four species showed high ammonia tolerance, compared to fishes and aquatic-breeding frogs. Both nest-dwelling larvae of Leptodactylus fragilis and late embryos of Hyalinobatrachium fleischmanni excreted urea, showing a plastic increase under dry conditions. These two species can develop the longest on land and urea excretion appears adaptive, preventing their exposure to potentially lethal levels of ammonia. Neither late embryos of Agalychnis callidryas nor nest-dwelling larvae of Engystomops pustulosus risked toxic ammonia levels under dry conditions, and neither excreted urea. Our results suggests that an early onset of urea excretion, its increase under dry conditions, and elevated ammonia tolerance, can all help prevent ammonia toxicity during terrestrial development. High ammonia represents a general risk for development that can be exacerbated as climate change increases dehydration risk for terrestrial-breeding frogs. It may also be a cue that elicits adaptive physiological responses during early development.
\end{abstract}

\section{Hosted file}

Ammonia\&Urea4Species-finalwithfigures.pdf available at https://authorea.com/users/429235/ articles/532965-reproductive-colonization-of-land-by-frogs-embryos-and-larvae-excreteurea-to-avoid-ammonia-toxicity 